\title{
Identification and verification of a longitudinal human driving model for collision warning and avoidance systems
}

\author{
Kangwon Lee and Huei Peng* \\ Department of Mechanical Engineering, University of Michigan, \\ Ann Arbor, MI 48109-2121, USA \\ E-mail: hpeng@umich.edu \\ *Corresponding author
}

\begin{abstract}
The main contribution of this paper is the identification of a human driving model based on field measured car-following data, and the verification of the model's performance in a microscopic traffic simulator. We first examined the SAVME database and obtained a well-defined set of data under closing-in and decelerating lead-vehicle scenarios, i.e. the transient manoeuvre starts with a large range and negative range rate toward the equilibrium point with proper range and zero range rate. Subsequently, the ICC FOT database is used to extract model parameters under normal highway driving conditions. These well-defined data sets are then used to test the flexibility of several existing driving models, i.e. the model parameters are tuned to fit these data. The Gipps model was found to be able to fit the highest number of manoeuvres, and the identified parameters are used to represent human-controlled vehicles, which are deterministic but have different attributes (aggressiveness, target speed, etc.). The Gipps model and the parameter sets are then implemented in a microscopic traffic simulator. Macroscopic and microscopic behaviours of these simulated human-controlled vehicles are presented.
\end{abstract}

Keywords: collision avoidance; forward collision warning; human driving model; microscopic traffic simulation.

Reference to this paper should be made as follows: Lee, K. and Peng, H. (2004) 'Identification and verification of a longitudinal human driving model for collision warning and avoidance systems', Int. J. Vehicle Autonomous Systems, Vol. 2, Nos. 1/2, pp.3-17.

Biographical notes: Kangwon Lee received the B.S. and M.S. degrees in Mechanical Engineering from Yonsei University, Seoul, South Korea, in 1993 and 1995, respectively. He obtained an M.S.E. degree in Electrical Engineering/Systems from the University of Michigan, Ann Arbor, in 2002, where he is currently working toward his Ph.D. degree in Mechanical Engineering. He worked for the Hyundai Motor Company from 1995 to 1998. His research interests are intelligent transportation systems with emphasis on adaptive cruise control systems and forward collision avoidance systems.

Huei Peng is currently an Associate Professor of the Department of Mechanical Engineering, and the Director of the Automotive Engineering Program at the University of Michigan, Ann Arbor. His research interests include adaptive control and optimal control, with emphasis on their applications to vehicular and transportation systems. He has also been an

Copyright (C) 2004 Inderscience Enterprises Ltd. 
active member of SAE and the ASME Dynamic System and Control Division, serving as the chair of the ASME DSCD Transportation Panel from 1995 to 1997. He is currently an Associate Editor for the IEEE/ASME Transactions on Mechatronics. He received the National Science Foundation (NSF) Career award in 1998.

\section{Introduction}

Forward Collision Warning systems refer to active safety devices designed to alert drivers in avoiding or reducing the severity of frontal crashes with another vehicle. This kind of active safety devices have received increasing attention in recent years mainly due to two reasons:

1 they target a major crash type ( $\sim 1 / 3$ of all road crashes in the US)

2 there is a significant overlap in underlying enabling technologies (sensors, dynamic models) with Adaptive Cruise Control (ACC) systems, which have been launched by many car companies in the last five years (DaimlerChrysler, Nissan, BMW, just to name a few).

It is natural to enhance working ACC systems with the additional forward Collision Warning $(\mathrm{CW})$ or Collision Avoidance functions (CA). Similar to ACC, many of the $\mathrm{CW} / \mathrm{CA}$ systems are intended to work in an autonomous fashion, in other words, to function properly without the cooperation of other vehicles, and infrastructures (including GPS).

Due to the difference in product segmentation (safety vs. comfort), however, many car companies are much more cautious in $\mathrm{CW} / \mathrm{CA}$ product design and launching. In addition to legal/liability concerns, this conservativeness is mainly due to the heavier reliance of $\mathrm{CW} / \mathrm{CA}$ systems on human follow-up actions. In the case of $\mathrm{ACC}$, the product was designed to keep the vehicle operating in regions where human intervention is normally unnecessary (safe time headway, small relative speed). Therefore, the characteristics of the human driver are relatively unimportant. In the case of $\mathrm{CW} / \mathrm{CA}$, however, the human drivers are always in control and could encroach on safe driving boundaries. Furthermore, they are responsible for reacting to the warning signal in a proper fashion solely or together with the automatic braking system. Therefore, designing a $\mathrm{CW} / \mathrm{CA}$ system that can accommodate a wide range of human characteristics is non-trivial.

One of the major difficulties posed by human characteristic variations lies in the engineering decision to balance system effectiveness against false/nuisance alarm rate. If the $\mathrm{CW} / \mathrm{CA}$ system is required to work with all human drivers, the resulting system would necessarily provide alerts in situations deemed non-threatening by a large percentage of drivers, and thus would be perceived as false/nuisance alarms. The high number of false/nuisance alarms may cause drivers to ignore future alerts and thus the system effectiveness could reduce substantially.

A better understanding of human characteristics is a necessary first step to address this user acceptance/performance issue for $\mathrm{CW} / \mathrm{CA}$ systems. Wiacek and Najm [1] studied the Driver/Vehicle Characteristics in Rear-End Pre-crash Scenarios 
based on the General Estimates System (GES) data. However, the results (due to the nature of the GES data) are mostly qualitative and descriptive and cannot be used to represent useful driver behaviour in accurate studies such as for computer simulations. Kiefer et al. [2] reported a detailed system minimum functional requirement study on a forward $\mathrm{CW}$ system. In term of human 'models', they reported results on a 'last-second braking test' to establish the timing and level of deceleration from human drivers. They also studied a set of driver behaviour parameters and their relationship to subjective rating on warning timing. Kuge et al. [3] proposed a driver behaviour recognition method based on Hidden Markov Models (HMM). The identified results are then used in a cognitive model of human drivers. Although their study was focused on lane-keeping/lane change behaviour, the methodology is potentially extendible to longitudinal speed/spacing control behaviour. Cheng and Fujioka [4] proposed a hierarchical driver model consisting of four layers - decision making, planning, manoeuvre, and action.

Since the early 1950s, many (non-CW/CA specific) human driving models have been proposed, and formed the basis of microscopic traffic simulation studies. Pipes suggested a linear follow-the-leader model [5]. This model assumes that the driver aims to accelerate the vehicle in proportion to the speed difference between the lead vehicle and the following vehicle. The proportional constant is termed the 'sensitivity' of the driver model. This desired acceleration is realised after a neuro-muscular delay. Chandler [6] identified the parameters (sensitivity and delay) of the Pipes model based on measured vehicle-following data. Gazis [7] extends the Pipes' model by assuming that the sensitivity of the follow-the-leader model is proportional to $\mathrm{m}^{\text {th }}$ power of velocity over $l^{\text {th }}$ power of range error. Newell [8] proposed a different model based on the assumption that human driver has a desired speed and a natural tendency to convergence to this desired speed in an exponential fashion. Tyler [9] formulated the human driver as a linear optimal controller, and the cost function being optimised is a quadratic function of range error and range rate error. Later Burnham [10] modified Tyler's model to include human reaction time and vehicle nonlinearities. Gipps [11] proposed a switching vehicle speed model based on two mutually exclusive considerations: to keep a safe distance from the lead vehicle and to converge to the desired free flow speed. In order to predict vehicle behaviour under both free and congested traffic with a single equation, Bando [12] devised a model named the 'optimal velocity model' which assumes a special basis function to describe human behaviour. She also identified these parameters from highway traffic data.

Various mathematical representations of human action were proposed in the models referred to above. However, few of them have been validated against real, vehicle level (microscopic) data. This is perhaps due to the fact that high quality vehicle driving data was hard to get until recently. In this paper, we will investigate many of the driver models discussed above and identify one that is flexible enough (i.e. with the most adaptable basis functions) to describe real human driving data most accurately. The driving data will be based on two databases recently constructed at the University of Michigan Transportation Research Institute (UMTRI): The SAVME database [13] and the ICC FOT database [14]. The identified driver model will then be implemented in a microscopic traffic simulator (UM ACCSIM) also developed at the University of Michigan [15]. 
The remainder of this paper is organised as follows: The two database systems and the driving data extraction procedure are described in Section 2. The six human driving models investigated in this paper are described in Section 3. Model correlation results are presented in Section 4. The UM ACCSIM and simulation results are described in Section 5. Finally, conclusions are drawn in Section 6.

\section{Driving data extraction}

The data used to select and verify the driving models in Section 3 are obtained from two sources: The SAVME database and the ICC FOT database. These two database systems are described in Sections 2.1 and 2.2, respectively. The data selection criteria and procedure are described in Section 2.3.

\subsection{SAVME database}

The SAVME (System for Assessment of the Vehicle Motion Environment, [13]) project aimed at documenting how human controlled vehicles move and position themselves in proximity to others during normal driving. A major deliverable from this project is an archival record of the behaviour of drivers on public roads, i.e. an authoritative form of 'truth data' documenting the conventional driving process. This database contains extremely valuable information for human model research such as the work reported in this paper.

The SAVME system collects digital video images from two roadside cameras at a $10 \mathrm{~Hz}$ sampling rate. The images are then processed to produce $10-\mathrm{Hz}$ data for each vehicle that passes through the selected road site. Both absolute and relative variables such as vehicle forward speed, position (longitudinal and lateral), range, range-rate, and azimuth angles are then computed. Through Kalman filtering, the dataset is then augmented with additional variables such as longitudinal acceleration, yaw rate, front wheel angle, lateral velocity, and heading angle.

Two batches of data (1996 and 1999) are available from roadside measurement of about 30,000 vehicles operating on a 5-lane arterial street in Ann Arbor, Michigan. These results are in the form of Microsoft ACCESS database of about $600 \mathrm{MB}$ each. Validation results show that spatial accuracies are within $2 \mathrm{ft}(0.6 \mathrm{~m})$ and the accuracy of velocity components is typically within $2 \mathrm{ft} / \mathrm{sec}(0.6 \mathrm{~m} / \mathrm{s})$. This error level is slightly worse than, but comparable to, that from other sensors such as radar, differential GPS, etc.

\subsection{ICC FOT database}

To observe the operability of the intelligent cruise control system in naturalistic use, the University of Michigan Transportation Research Institute performed an Intelligent Cruise Control Field Operational Test [14]. Volunteer drivers numbering 108 participated in the test for two to five weeks each with their driving behaviour and vehicle motion faithfully recorded throughout the period of the operation (1-2 weeks each). Infrared laser sensors with a steered $2^{\circ}$-wide-133 m-long sweep beam and $7^{\circ}$-wide- $32 \mathrm{~m}$-long 'cut-in' beam were used to measure the range and 
range rate from the lead vehicle when one is present. The variables recorded include time, date, range, range rate, velocity, lead vehicle speed, throttle level, and ICC operation mode. The ICC could be set to either 'off', 'standby', 'conventional' or 'intelligent' modes. The results are a collection of more than $100 \mathrm{CDs}$ of data. In this paper, a small subset of the data in 'off' or 'standby' mode (i.e. human driving mode) were extracted and used.

\subsection{Data selection}

Originally, both SAVME and ICC FOT data were stored in the Microsoft Access database format. MS Access query commands, Visual Basic programmes and MATLAB scripts were used to extract vehicle interaction data and prepare them in a suitable form for the driver model simulations (see Figure 1). Due to the difference in the nature of the variables of these two databases, the data selection and processing procedures are different. The procedure and criteria are described separately below.

Figure 1 Data processing block diagram

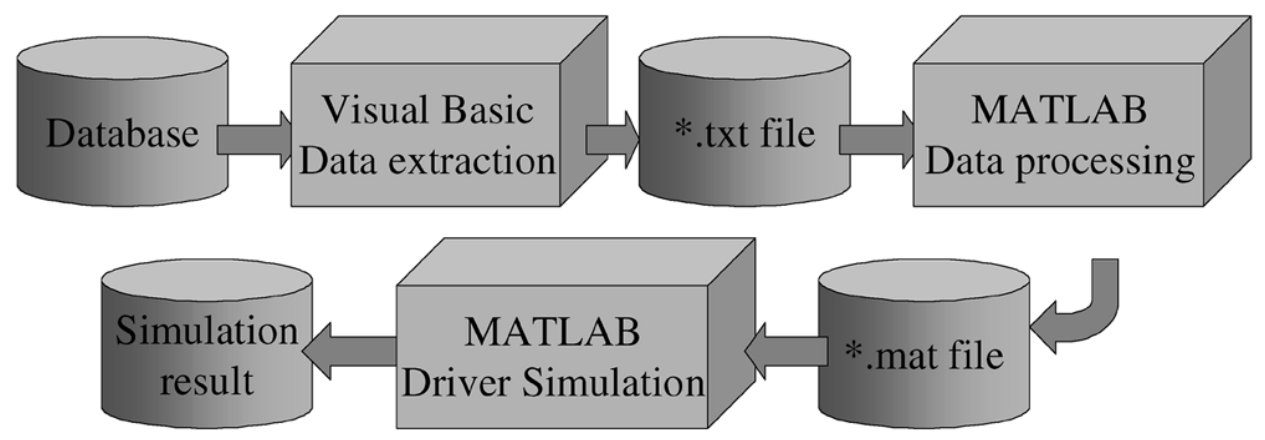

\section{SAVME}

\subsection{Vehicle (leader-follower) pair extraction}

In the SAVME database, absolute $x-y$ locations of all the vehicles inside the camera covered region are recorded. To focus on the car-following cases, leader-follower pairs in the same lane need to be identified and all relevant variables (range, range rate, etc.) are calculated. We also eliminate all cases when the leader or the follower executes a lane change.

The above criteria are all realised by set proper query commands. To give an example on how these data-selection criteria can be realised, we will describe the 'no-lane-change' criterion below. To find the vehicle pairs without any lane change, maximum and minimum of lane number of each vehicle was calculated. If the maximum and minimum lane numbers are different, that indicates that a lane change has occurred and thus the data will not be selected. 


\subsection{Data quality calculation}

A Visual Basic programme was written to calculate the raw data quality for each vehicle: root-mean-square error between the numerical differentiation of un-filtered position and filtered velocity and root-mean-square error between the numerical differentiation of filtered position and filtered velocity. Data from vehicles whose first RMS error was larger than $6 \mathrm{~m} / \mathrm{sec}$ or second RMS error larger than $0.3 \mathrm{~m} / \mathrm{sec}$ were rejected.

\subsection{Select cases of interest}

Of all the vehicle pairs that satisfy the error criteria stated above, data points with time to impact less than 11 seconds were extracted. These data are judged to be 'interesting' because they are most relevant for future collision warning and collision control system calibration and verification. For example, the behaviour of the following-vehicle in these cases are assumed to be what human drivers felt comfortable with and thus will be used as templates for $\mathrm{CW} / \mathrm{CA}$ designs. Among all the eligible data, the 100 longest cases were selected for the driver model evaluations to be described in Section 3 .

\section{ICC-FOT}

A Visual Basic programme was written to extract data from the ICC-FOT database that satisfy the following conditions:

- the ICC mode is either off or standby (Human driving)

- a lead-vehicle was detected by the range sensor

- vehicle speed is always larger than 0

- the same lead-vehicle throughout the vehicle interaction.

The eligible data were sorted and the 100 longest car-following cases were identified and used to represent 'driver population' under normal driving conditions. This driver population will be used in the traffic simulator as 'background traffic' for future $\mathrm{CW} / \mathrm{CA}$ studies. The selected SAVME and ICCFOT data are plotted in Figure 2. 
Figure 2 Range rate-range distribution, SAVME \& ICCFOT data

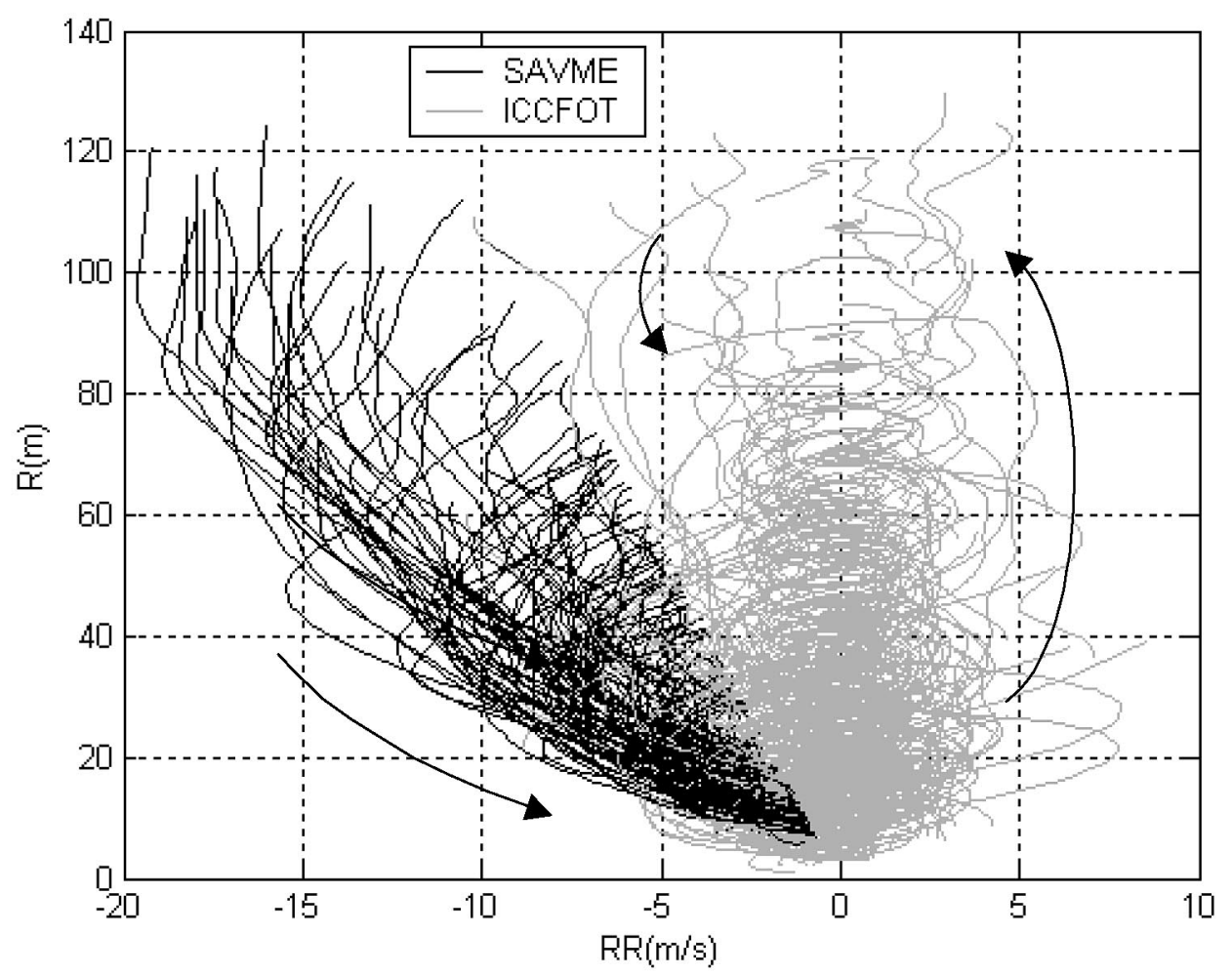

\section{Driving models evaluation}

\subsection{Correlation study}

The real human driving data extracted from the two database systems can be used to evaluate existing driving models to see whether they use 'basis functions' or templates that are rich enough and 'degrees of freedom' that are flexible enough to describe most of the real driving datasets with acceptable accuracy. In the evaluation, we will allow all the 'degrees of freedom' of the models (free parameters) to change from one dataset to the next, as long as the variations are allowed in the proposed model. All the models evaluated in this section have been reviewed in Section 1, with key references. Interested readers can find out the details from those publications. The model equations and degrees of freedom (DOF) are summarised in Table 1. It can be seen that the DOF of these models vary between two and five, and thus the comparison between them may not be completely fair (and is not the purpose of this study). The purpose of this evaluation study is to see whether any model is flexible enough, and if so, to build a look-up table detailing the distribution of its parameters, which can then be used to build a vehicle population in traffic simulators to represent real human drivers with different personalities. 
Table 1 Summary of longitudinal driver models

\begin{tabular}{|c|c|c|}
\hline Model & $D O F$ & Equation \\
\hline $\begin{array}{l}\text { Linear } \\
\text { follow- } \\
\text { the-leader }\end{array}$ & $K, \tau$ & $\dot{V}_{F}=a_{c}=K e^{-\tau s}\left(V_{L}-V_{F}\right)$ \\
\hline Newell & $\begin{array}{l}V_{j 0} \\
\lambda_{j} \\
d_{j}, \Delta\end{array}$ & $v_{j}(t)=V_{j}\left[1-\exp \left\{-\lambda_{j} V_{j}^{-1}\left[x_{j-1}(t-\Delta)-x_{j}(t-\Delta)-d_{j}\right]\right\}\right]$ \\
\hline $\begin{array}{l}\text { Nonlinear } \\
\text { follow- } \\
\text { the-leader }\end{array}$ & $\begin{array}{l}C \\
\tau\end{array}$ & $\ddot{x}_{n+1}(t+T)=\frac{c \dot{x}_{n+1}}{\left(x_{n}-x_{n+1}\right)}\left[\dot{x}_{n}(t)-\dot{x}_{n+1}(t)\right]$ \\
\hline $\begin{array}{l}\text { Linear } \\
\text { optimal } \\
\text { control }\end{array}$ & $\begin{array}{l}C_{V} \\
C_{S} \\
C_{C}\end{array}$ & $u(t)=C_{v}\left(V_{L}-V_{F}\right)+C_{S}\left[\left(S_{L}-S_{F}\right)-C_{c} \cdot V_{F}\right]$ \\
\hline & & $\left(v_{n}(t)+2.5 a_{n} \tau\left(1-\frac{v_{n}(t)}{V_{n}}\right) \sqrt{0.025+\frac{v_{n}(t)}{V_{n}}}\right.$ \\
\hline Gipps & $\begin{array}{l}a_{n} \\
V_{n}\end{array}$ & $v_{n}(t+\tau)=\min \left\{b_{n} \tau+\sqrt{b_{n}^{2} \tau^{2}-b_{n}\left[2\left[x_{n-1}(t)-s_{n-1}-x_{n}(t)\right]-v_{n}(t) \tau-\frac{v_{n-1}(t)^{2}}{\hat{b}}\right]}\right.$ \\
\hline $\begin{array}{l}\text { Optimal } \\
\text { velocity }\end{array}$ & $\begin{array}{l}a, \\
V_{0} \\
m \\
b_{c}, b_{f}\end{array}$ & $\begin{aligned} \ddot{x}_{n} & =a\left[V(\Delta x)-\dot{x}_{n}\right]=2.0\left[V(\Delta x)-\dot{x}_{n}\right] \\
V(\Delta x) & =V_{0}\left[\tanh m\left(\Delta x-b_{f}\right)-\tanh m\left(b_{c}-b_{f}\right)\right]\end{aligned}$ \\
\hline
\end{tabular}

The evaluation procedure is as follows: First, we use the MATLAB command fminsearch() to identify the values of all the free parameters of a model that results in the smallest overall error between the predicted vehicle speed, and the true vehicle speed as indicated by the SAVME and ICC FOT results. Multiple initial conditions were tried to prevent a numerical trap at a local minimum. We repeat this optimisation procedure throughout the 100 approaching cases extracted from the SAVME database and the 100 car-following cases extracted from the ICC FOT datasets, allowing a new set of values for the free parameters for each new manoeuvre (new driver or new engagement). Once all the optimal model parameters were identified, we calculate the correlation between the predicted and the true vehicle speed. If the model is rich enough and flexible enough to accommodate a particular dataset, it will be able to find a set of values for the free parameters that achieves high correlation with the dataset. It is important to remember that in many cases the driver may not have focused on the driving task with full attention and thus it might be hard to make any sense out of the driving behaviour demonstrated. Therefore, we did not expect to achieve an extremely high correlation. It is also important to point out that several of the models predicted vehicle acceleration rather than speed. For 
those models, we will add an integrator to convert the model output to vehicle speed. This universal model output is important to ensure a fair comparison. The correlation results for the six driving models are shown in Figures 3 and 4.

Figure 3 Correlation results for six driving models (ICC FOT data)

\section{Correlation distribution- Velocity models and data}

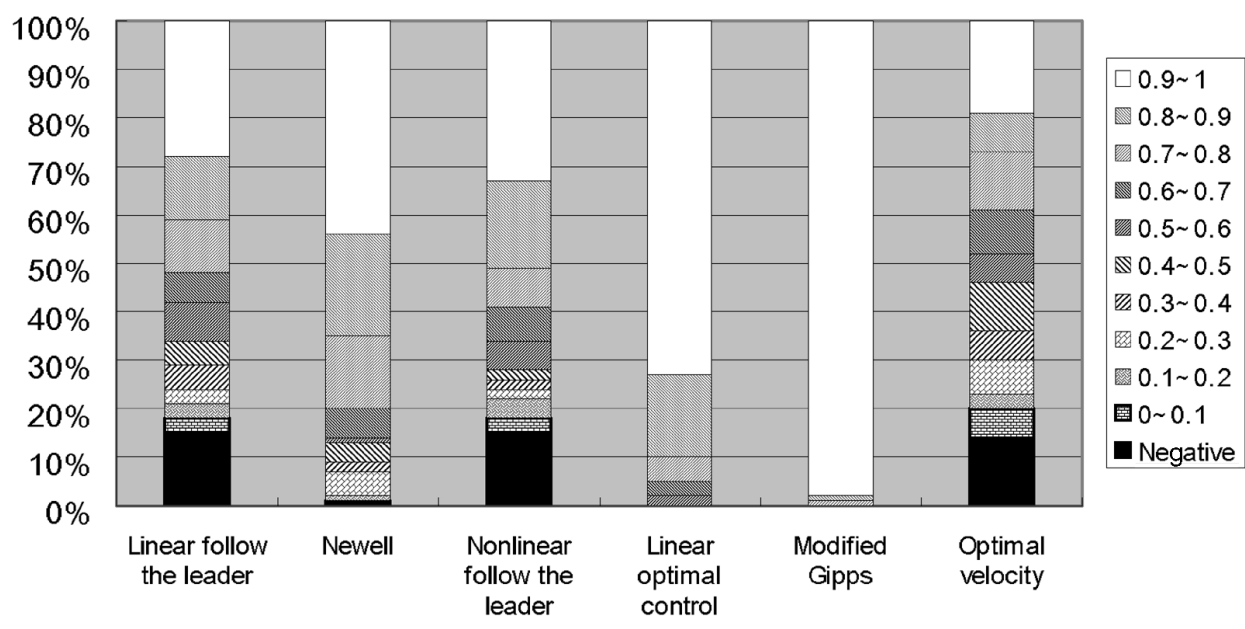

Figure 4 Correlation results for six driving models (SAVME data)

Correlation distribution- Velocity models and data

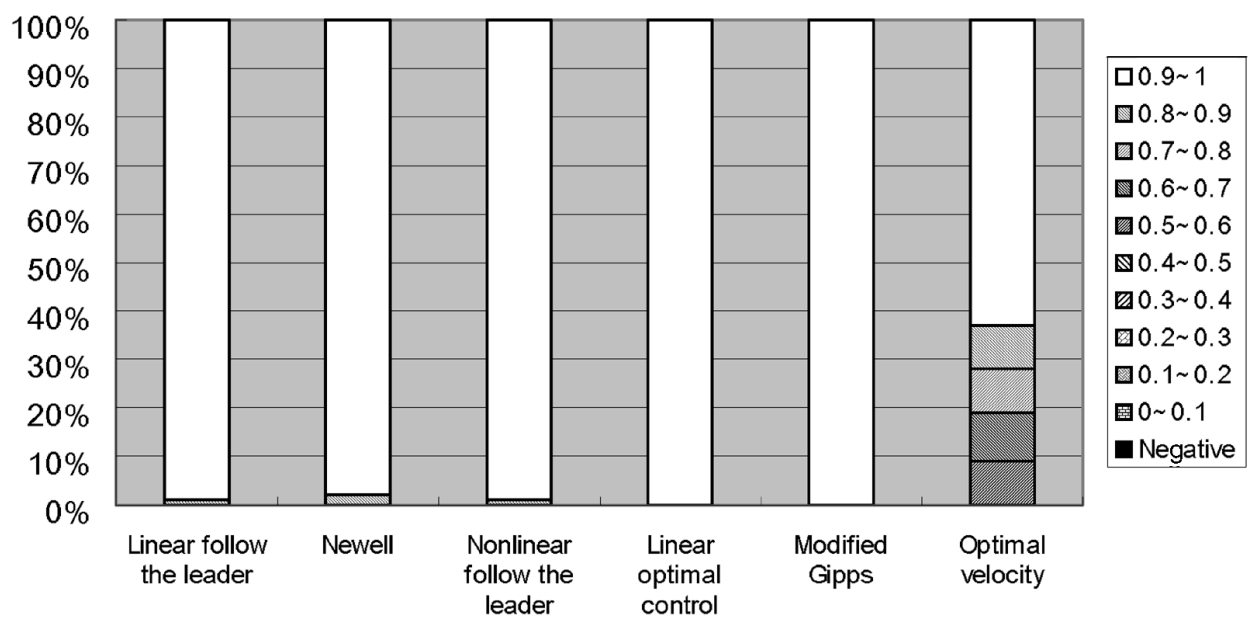


It can be seen that among the six models studied, the modified Gipps model demonstrated the highest flexibility. It has high $(>0.9)$ correlation with $90 \%$ of the datasets from both databases. We have no basis to judge what percentage of the datasets are 'good' data, in the sense that the vehicle reflects the behaviour of a safety-conscious driver. For the ICC FOT data, many of the selected datasets last for 100 seconds or longer. Therefore, the human drivers may not be fully engaged in the driving task throughout the whole manoeuvre. The SAVME data, on the other hand, last for no more than 15 seconds for most of the cases, and are pretty much in the approaching phase. Furthermore, the SAVME data are manually selected from a very large population (100 out of 30,000), and thus are almost always 'good' data. We conclude from the correlation study that most models can do a reasonable job in fitting the 'good' data, but only the Gipps model survives the 'torture test', which might include some 'bad' data. The Gipps model is selected because of this higher level of robustness, as well as the fact that it has only two DOF, and thus is easier to implement. In addition, when the model parameters are selected from the ICCFOT database, the behaviour of the vehicle is mostly cruising and thus could be used to represent 'background traffic', for the purpose of the evaluation of active safety control systems such as Adaptive Cruise Control or CW/CA systems. We will discuss the modified Gipps model and its identified DOF in details below.

\subsection{Gipps model}

Analysis results on the original Gipps model suggest that the steady-state time headway is too small, and some modifications are necessary. The details of the modification will be published in a future paper because of their length. The suggested Gipps model is given below

$$
v_{n}(t+\tau)=\min \left\{\begin{array}{c}
v_{n}(t)+2.5 a_{n} \tau\left(1-\frac{v_{n}(t)}{V_{n}}\right) \sqrt{0.025+\frac{v_{n}(t)}{V_{n}}} \\
b_{n} \tau+\sqrt{b_{n}^{2} \tau^{2}-b_{n}\left[2\left[x_{n-1}(t)-s_{n-1}-x_{n}(t)-v_{n}(t) \tau\right]-\frac{v_{n-1}(t)^{2}}{\hat{b}}\right]} .
\end{array}\right.
$$

The definition of the model variables is given in Table 2. The model output switches between two equations. The first equation represents the driver's desire to achieve the desired (free-flow) speed. The second equation estimates the safe range that needs to be maintained under hard-braking by the lead vehicle. We follow the original Gipps proposition and do not treat $b_{n}, \hat{b}$ and $\tau$ as free parameters. Rather, they are constrained by the following equations: $b_{n}=-2.0 a_{n}, \hat{b}=\min \left(-3.0,\left(b_{n}-3.0\right) / 2\right)$ and $\tau=2 / 3$ second. We further assume that the effective vehicle size $s_{n}=4 \mathrm{~m}$ and thus is not a free variable. 
Table 2 Parameters of the modified Gipps model

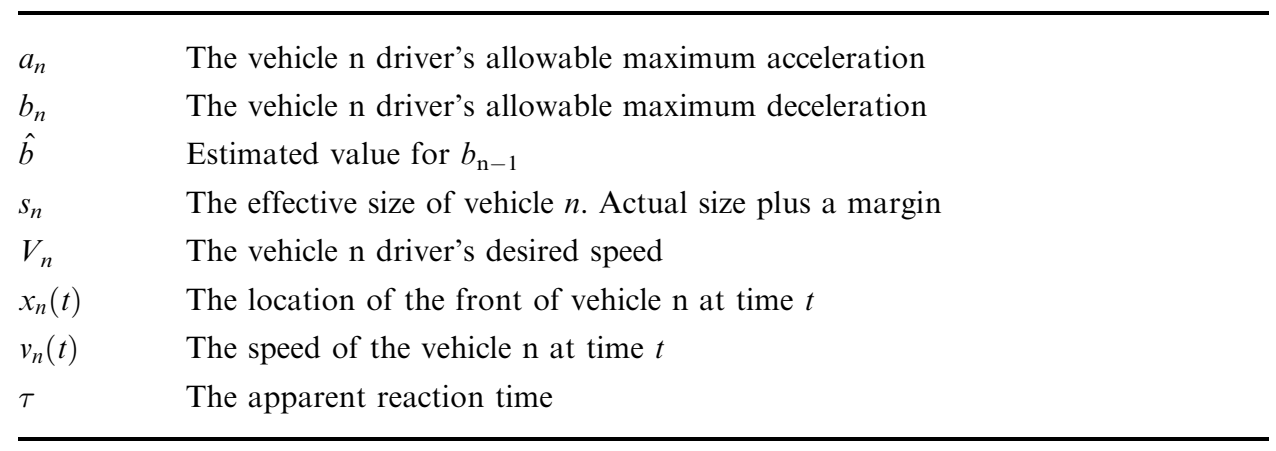

When only $a_{n}$ and $V_{n}$ are treated as free parameters, and the simplex method is used to fit the data from the ICC FOT database (with about 50 hours worth of driving data), the distribution of parameters is found to be that shown in Figure 5. This list of parameter values represents about 100 'drivers' and will be used in the next section to represent vehicles with different personalities.

Figure 5 Gipps model parameter distributions from ICCFOT data

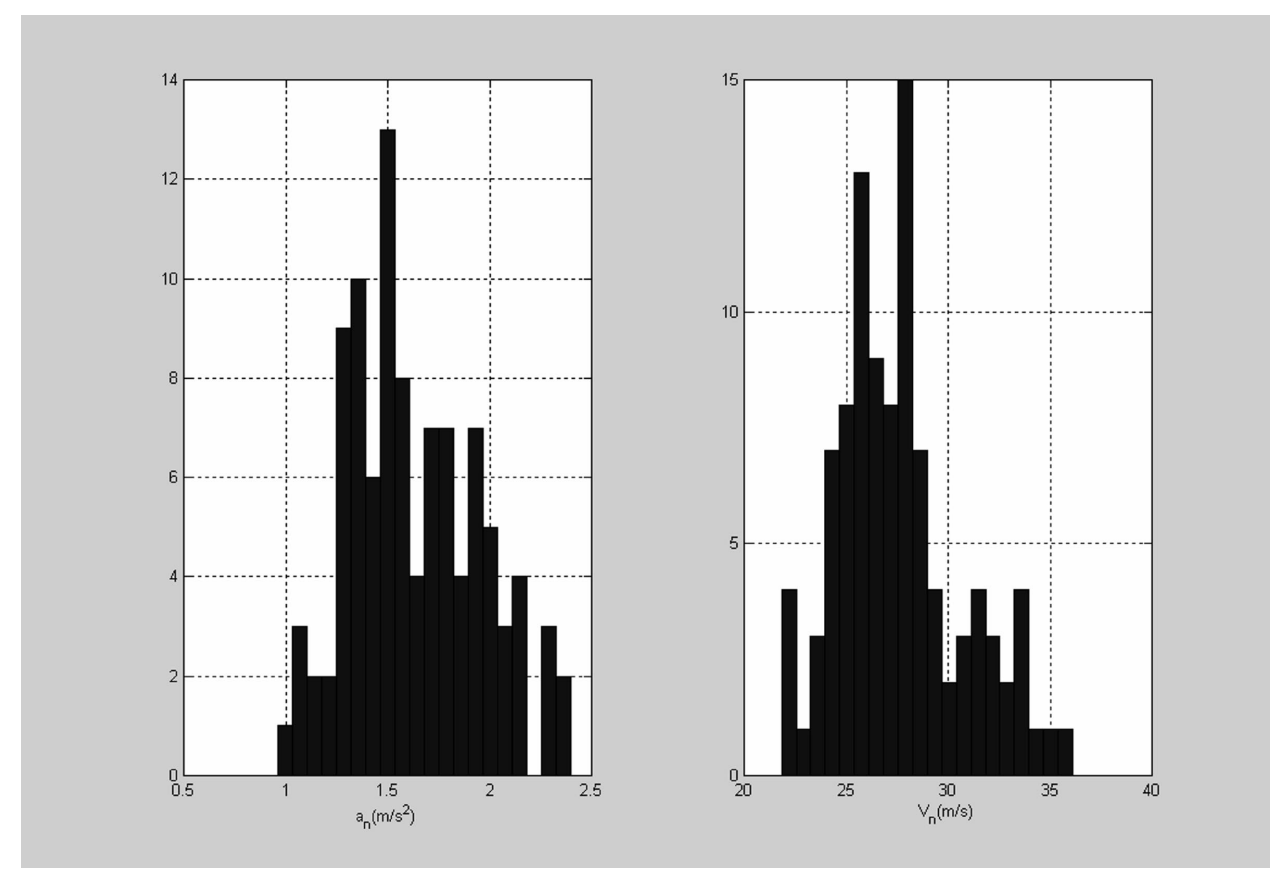




\section{Microscopic traffic simulation}

In recent years, the number of microscopic models and simulation tools has increased dramatically. These models, as opposed to their macroscopic counterparts, keep track of the motion of individual vehicles and are more suitable for the study of active safety devices such as $\mathrm{CW} / \mathrm{CA}$ systems. Examples of recent microscopic models include AIMSUN, SmartAHS, TRANSIMS, CORSIM, etc.

One of the key benefits of these microscopic models over fluid-based macroscopic models is the possibility of recognising each vehicle/driver's 'personality'. Therefore, more accurate evaluation of an individual vehicle's response in a realistic traffic environment is possible. Drivers' behaviour can be described either by certain car-following/lane change models or by a set of if-then rules. These models or rules generally dictate the lane location, forward speed or acceleration of each vehicle at each sampling time, based on a distribution of driver and vehicle parameters.

A microscopic simulator (UM-ACCSIM) was developed at the University of Michigan to evaluate the performance of ACC/CW-CA systems [15]. This software simulates and records the motions of each vehicle operating on a 2-lane circular track, and can produce many important microscopic and macroscopic outputs. The car-following, headway, average speed and lane change models are all constructed based on the statistical analysis of human drivers' characteristics from field measurement work [14]. Due to the existence of two lanes, frequent lane changes, and thus lead vehicle speed perturbations, occur. Therefore, it provides a naturalistic environment for the analysis of vehicles equipped with ACC and CW/CA systems.

A major drawback of this simulator [15] is the fact that it uses Pipes model, and that all vehicles have the same model parameters (personalities) in terms of its dynamic speed control behaviour. By introducing the Gipps model into this simulator, all aspects of human driving models used in controlling individual vehicle motions are now based on statistical driver behaviour data, thus completing its status of being a empirical data-based simulator. If all the behaviour models describe human-controlled vehicles with high enough fidelity, we expect the overall simulator to demonstrate many characteristics exhibited by human controlled vehicles/traffic, such as shock wave, traffic flow/density relationship, etc.

In the following, simulations of the enhanced UM-ACCSIM software were performed at controlled traffic density. The traffic density is said to be $100 \%$ if, on the averaged sense, the vehicles are allowed to drive at their desired speed (mean $\sim 23 \mathrm{~m} / \mathrm{sec}$ ) and keep their desired time headway (mean $\sim 1.5 \mathrm{sec}$ ) but otherwise fully occupy both lanes of the roadway. If the vehicle population is lower (higher), the vehicle density is said to be less (more) than $100 \%$. When the traffic density is low, no shock wave is observed in the simulations, probably because the denser traffic built up behind the shockwave can be relieved through lane changes. When the traffic density is high, lane changes become rare because empty spots are harder to come by. The behaviour is, therefore closer to that of a single-lane. In this case, shockwaves, i.e. propagation of slow-travelling traffic upstream of the highway, becomes quite noticeable (Figure 6). This figure also points out a potential need for future improvement: when the shockwave occurs at very low vehicle speed, vehicles will usually exhibit stop-and-go behaviour, and the shockwave grows in size. The shockwaves observed in the simulations, however, smooth out gradually with time. It 
seems the vehicle characteristics we observed at a higher speed in the ICCFOT database cannot accurately describe behaviour at low vehicle speed. If the intent is to use this simulator for simulations down to very low vehicle speeds, additional factors need to be introduced to reflect the fact that vehicles are more sluggish at a lower speed.

Figure 6 Observed shockwave behaviour from UM-ACCSIM simulations

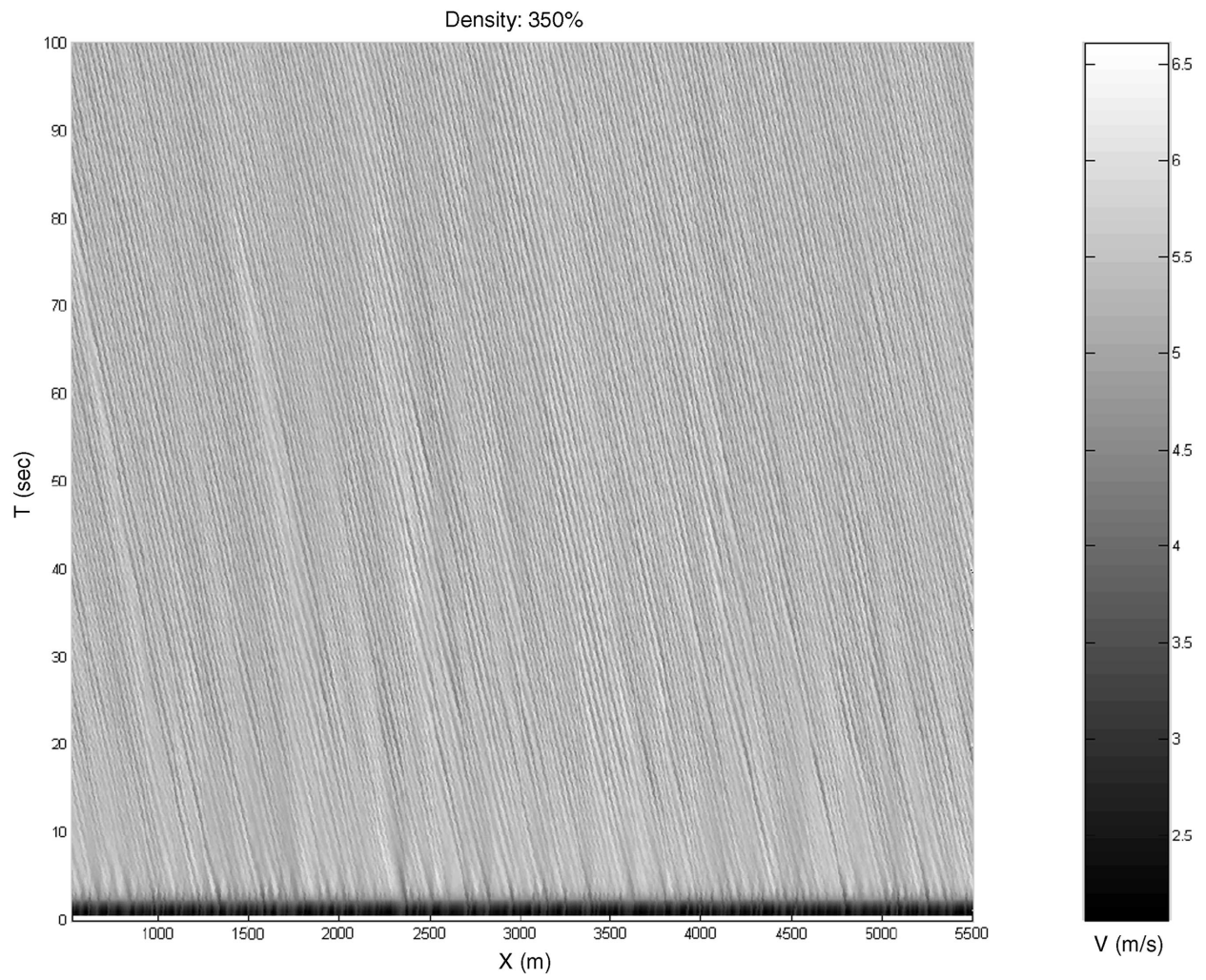

When we start from lower traffic density and keep adding vehicles for subsequent simulations, and calculate overall traffic flow (over a period of 60 minutes), it can be seen from Figures 7 and 8 that the traffic flow peaks at about 1,800 vehicles/lane/hr. The average speed continuously reduces when density increases. In both figures, we have shown both simulated results, as well as observed traffic data [16]. The boundaries of the so-called 'aggressive drivers' and 'conservative drivers' shown in Figure 7 were imagined at the upper and lower limits from the observed traffic data reported in [17]. It is clear that the simulated results are qualitatively similar to observed traffic data. Again, at high density and low speed ( $>70$ vehicles $/ \mathrm{km}$ ), the simulated results are more 'aggressive' than observed traffic. Additional factors to increase the sluggishness of vehicles might be needed. 
Figure 7 Flow rate vs density

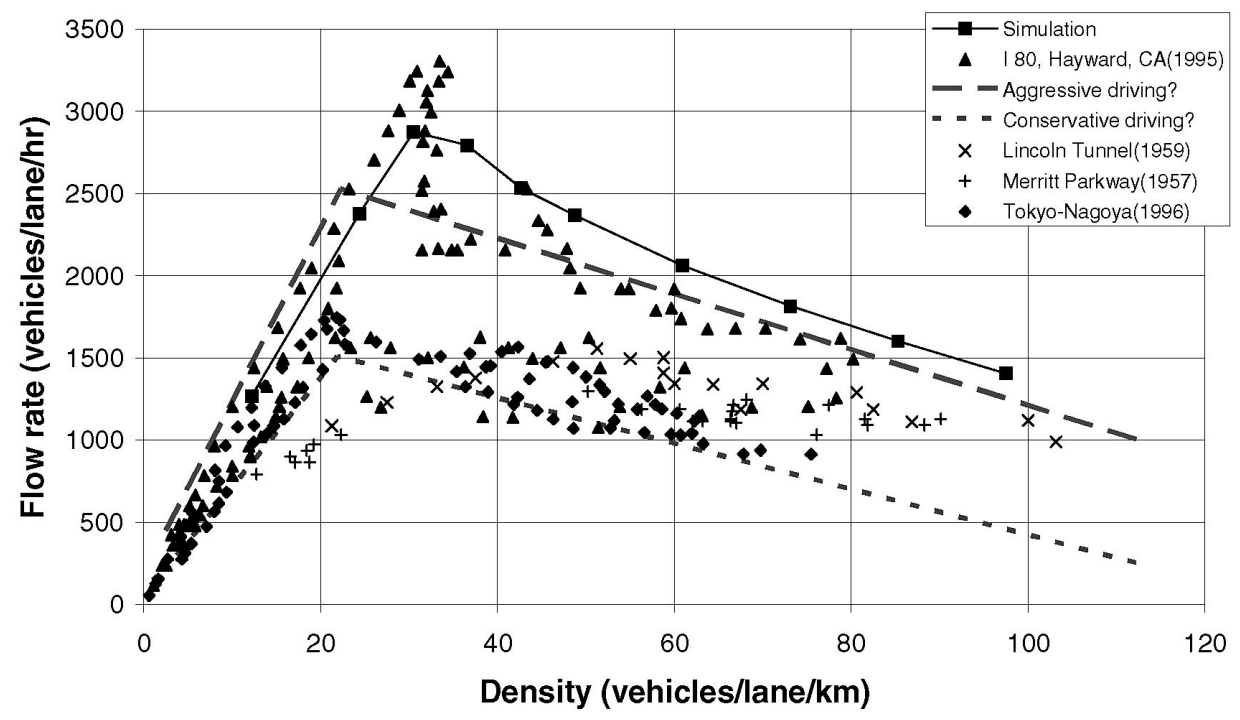

Figure 8 Average speed vs density

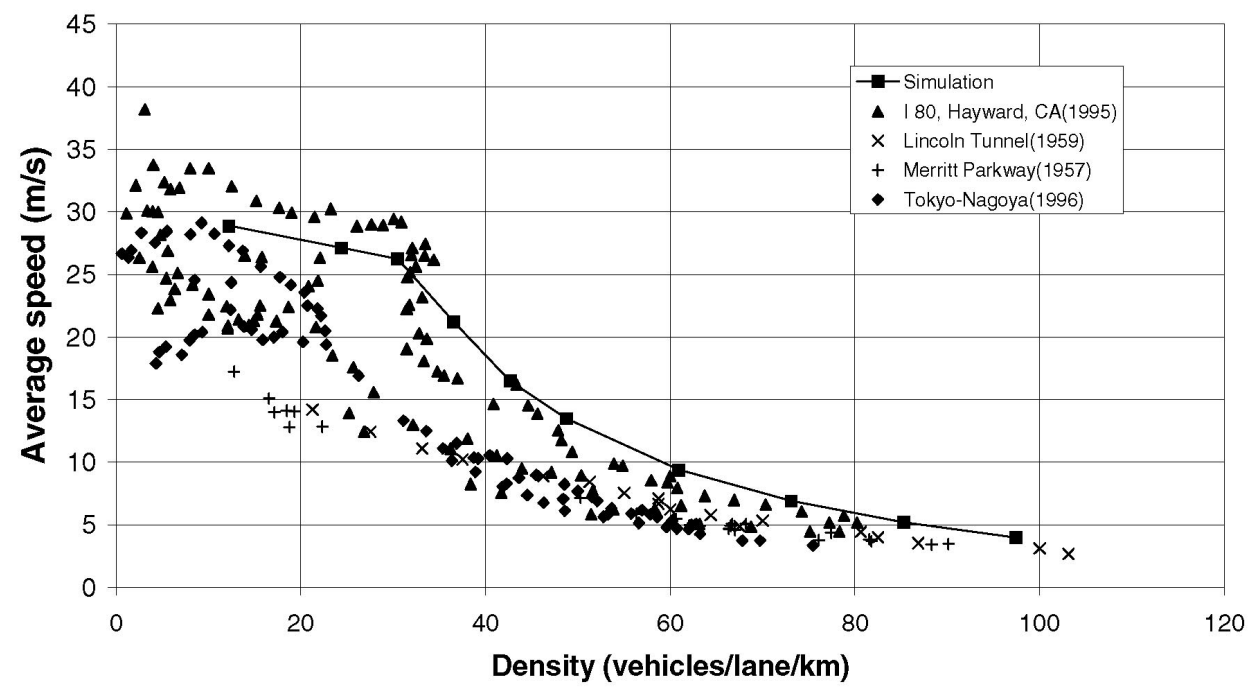

\section{Conclusions}

In this paper, we have reported the identification of a driver forward speed control model that is suitable for the design and evaluation of collision warning and collision avoidance $(\mathrm{CW} / \mathrm{CA})$ systems. We found that many of the existing driving models can do a reasonable job when they are evaluated only for the 'approaching' cases, i.e. 
when the host vehicle is slowing down from behind a slower lead vehicle. When the vehicle is in general car-following mode, the driver may not be paying full attention to the driving task. For these more general, and possibly lower-quality data, only the modified Gipps model works satisfactorily, with more than $90 \%$ of the test data accurately fitted. We implemented the modified Gipps model and found that the simulated traffic exhibits many of the microscopic and macroscopic characteristics observed in real traffic. We will use the simulator for future $\mathrm{CW} / \mathrm{CA}$ system design and verifications.

\section{References}

1 Wiacek, C.J. and Najm, W.G. (1999) 'Driver/vehicle characteristics in rear-end precrash scenarios based on the general estimates system (GES)', SAE Paper 1999-01-0817.

2 Kiefer, R., Leblanc, D., Palmer, M., Salinger, J., Deering, R. and Shulman, M. (1999) 'Development and validation of functional definitions and evaluation procedures for collision warning/avoidance systems', NHTSA Technical Report, DOT HS 808964.

3 Kuge, N. et al. (2000) 'A driver behaviour recognition method based on a driver model framework', SAE Paper 2000-01-0349.

4 Cheng, B. and Fujioka, T. (1997) 'A hierarchical driver model', IEEE Conference on Intelligent Transportation System, pp.960-965.

5 Pipes, L.A. (1953) 'An operational analysis of traffic dynamics', Journal of Applied Physics, Vol. 24, pp.271-281.

6 Chandler, F.E., Herman, R. and Montroll, E.W. (1958) 'Traffic dynamics: studies in car following', Operations Research, Vol. 6, pp.165-184.

7 Gazis, D.C., Herman, R. and Rothery, R.W. (1961) 'Nonlinear follow-the-leader models of traffic flow', Operations Research, Vol. 9, pp.545-566.

8 Newell, G.F. (1961) 'Nonlinear effects in the dynamics of car following', Ops. Res., Vol. 9, pp.209-229.

9 Tyler, J.S., (1964) 'The characteristics of model following systems as synthesises by optimal control', IEEE Trans. Automatic Control, Vol. AC-9, pp.485-498.

10 Burnham, G.O., Seo, J. and Bekey, G. A. (1974) 'Identification of human driver models in car following', IEEE Trans. on Automatic Control, Vol. AC-19, pp.911-915.

11 Gipps, P.G. (1981) 'A behavioural car-following model for computer simulation', Transportation Research B, Vol. 15B, pp.105-111.

12 Bando, M. et al. (1995) 'Phenomenological study of dynamical model of traffic flow', Journal De Physique, I, Vol. 5, pp.1389-1399.

13 Ervin, R. et al. (2000) 'System for assessment of the vehicle motion environment (SAVME)', University of Michigan Transportation Research Institute, UMTRI-2000-21-1, August.

14 Fancher, P. et al. (1998) 'Intelligent cruise control field operational test', DOT HS 808 849, May.

15 Liang, C. and Peng, H. (2000) 'String stability analysis of adaptive cruise controlled vehicles', JSME International Journal Series C, Vol. 43, No. 3, September, pp.671-677.

16 Gartner, N.H. et al. (Eds.) (1997) Monograph on Traffic Flow Theory, Oak Ridge, Tennessee, USA: Oak Ridge National Laboratory (URL: http://stargate.ornl.gov/trb/ tft.html).

17 Kockelman, K.M. (2001) 'Modeling traffic's flow-density relation: accommodation of multiple flow regimes and traveler types', Transportation, Vol. 28, No. 4, pp.363-374. 